Aktuelle Beiträge zum öffentlichen Recht

herausgegeben von:

Rolf Gröschner

Martin Morlok

Helmuth Schulze-Fielitz

Band 2 


\section{Das Recht auf Umweltinformation}

unter besonderer Berücksichtigung

des Schutzes von Betriebs- und

Geschäftsgeheimnissen

und der Paragraphen 207-227 UGB-KomE

Max Koebke

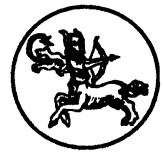

Centaurus Verlag \& Media UG 1999 
Der Autor, geb. 1971, studierte Rechtswissenschaften. Er promovierte 1998 an der Universität Würzburg. Er ist derzeit als Rechtsreferendar tätig.

Die Deutsche Bibliothek - CIP-Einheitsaufnahme

\section{Koebke, Max:}

Das Recht auf Umweltinformation unter besonderer Berücksichtigung

des Schutzes von Betriebs- und Geschäftsgeheimnissen und der

Paragraphen 207-227 UGB-KomE / Max Koebke. - Pfaffenweiler :

Centaurus-Verl.-Ges., 1999

(Aktuelle Beiträge zum öffentlichen Recht ; Bd. 2)

Zugl.: Würzburg, Univ., Diss., 1998

ISBN 978-3-8255-0264-5

DOI $10.1007 / 978-3-86226-323-3$

\section{ISSN 0941-4363}

Alle Rechte, insbesondere das Recht der Vervielfältigung und Verbreitung sowie der Übersetzung, vorbehalten. Kein Teil des Werkes darf in irgendeiner Form (durch Fotokopie, Mikrofilm oder ein anderes Verfahren) ohne schriftliche Genehmigung des Verlages reproduzient oder unter Verwendung elektronischer Systeme verarbeitet, vervielfältigt oder verbreitet werden.

(C) CENTAURUS-Verlagsgesellschaft mit beschränkter Haftung, Pfaffenweiler 1999

Satz: Vorlage des Autors 


\section{Inhaltsverzeichnis}

Literaturverzeichnis IX

Einleitung 1

1. Kapitel Rechtssystematische Grundlagen eines Informationsanspruchs 3

I. Ausgestaltung von Informationsansprüchen $\quad 3$

1. Kein Zugangsrecht 5

2. Situationsabhängiger Zugang $\quad 10$

a) Die Aktenöffentlichkeit im Verwaltungsverfahren nach $\S \S 29,30$ VwVfG 11

(1) Bedeutung der Aktenöffentlichkeit im Verfahren 13

(2) Anspruchsinhalt $\quad 14$

b) Öffentliche Auslegung im (vorgezogenen) Verwaltungsverfahren $\quad 18$

(1) Die Verwaltungsöffentlichkeit des Bundesimmissionsschutzgesetzes 18

(2) Informationsrechte im Atomrecht 22

(3) Informationsrechte nach den Grundsätzen der Umweltverträglichkeitsprüfung 23

(4) Informationsrechte im Gentechnikrecht 25

c) Das Akteneinsichtsrecht im (verwaltungs-) gerichtlichen Verfahren 26

d) Zwischenergebnis für verfahrensbezogene Informationsansprüche 27

e) Das Einsichtsrecht aufgrund persönlichen Bezugs der Informationen 28

(1) Der Auskunftsanspruch nach dem Bundesdatenschutzgesetz 29

(2) Das Unterlageneinsichtsrecht nach dem Stasiunterlagengesetz 31

(3) Zwischenergebnis für Einsichtsrechte aufgrund persönlichen Bezugs der Informationen $\quad 33$

3. Freier Zugang (am Beispiel der Umweltinformationen) 33

II. Amtsgeheimnis und Informationsfreiheit im System der Staatsprinzipien und Freiheitsrechte 37

1. Informationsfreiheit des Art. 5 Abs. 1 S. 1 Hs. 2 GG 37

2. Objektiv-rechtliche Dimension des Art. 5 Abs. 1 GG 39

3. Demokratieprinzip $\quad 42$

III. Weiterentwicklung im Kommissionsentwurf zu einem Umweltgesetzbuch 45

1. Die umfassende Informationsverwaltung 46

2. Die Umweltinformationsrichtlinie im Kommissionsentwurf 47

3. Demokratische Aspekte im Kommissionsentwurf 48

$\begin{array}{ll}\text { IV. Informationsrechte in anderen Staaten } & 48\end{array}$

1. USA - Freedom of Information Act

a) Allgemeines 51

b) Entstehungsgeschichte 52

c) Verfassungsrechtliche Stellung 54

d) Ausnahmen von der Informationspflicht, insbesondere der Schutz des Betriebs- und Geschäftsgeheimnisses $\quad 55$

2. Nordeuropäische Länder 57 
a) Schweden - Tryckfrihetsförordning

(1) Allgemeines $\quad 57$

(2) Verfassungsgeschichtliche Entwicklung 59

(3) Verfassungsrechtliche Stellung $\quad 59$

(4) Ausnahmen von den Informationsrechten, insbesondere der Schutz des Betriebs- und

$\begin{array}{ll}\text { Geschäftsgeheimnisses } & 60\end{array}$

$\begin{array}{ll}\text { b) Norwegen } & 61\end{array}$

c) Finnland $\quad 61$

$\begin{array}{ll}\text { d) Dänemark } & 61\end{array}$

$\begin{array}{ll}\text { 3. Benelux-Staaten } & 62\end{array}$

a) Niederlande $\quad 62$

b) Belgien 64

c) Luxemburg $\quad 65$

4. Frankreich - Loi sur l'accès aux documents administratifs (Loi no. 78-753) 65

a) Allgemeines $\quad 65$

b) Verfassungsgeschichtlicher Hintergrund 66

c) Grenzen des Anspruchs 67

5. Großbritannien und Irland: Vom Official Secrets Act zum Freedom of Information Act 68

a) Vereinigtes Königreich $\quad 68$

(1) Das traditionelle Geheimhaltungsprinzip $\quad 68$

(2) Änderungen nach dem Regierungswechsel zur Labour Party 70

(3) Code of Practice $\quad 70$

b) Irland $\quad 71$

$\begin{array}{ll}\text { 6. Österreich } & 72\end{array}$

7. Südeuropäische Staaten $\quad 72$

a) Italien $\quad 72$

b) Griechenland $\quad 72$

c) Portugal $\quad 73$

$\begin{array}{ll}\text { d) Spanien } & 74\end{array}$

$\begin{array}{ll}\text { 8. Europäische Institutionen } & 74\end{array}$

9. Zusammenfassung $\quad 81$

a) Bedeutung von Demokratie als Begründung für Zugangsrechte 82

b) Einzelheiten zum Zugangsrecht $\quad 83$

c) Grenzen des Anspruchs $\quad 84$

2. Kapitel Deutsche und Europäische Zwecke und Zielsetzungen des Anspruches auf Umweltinformationen $\quad 86$

I. Verbesserung des Umweltschutzes $\quad 86$

1. Erhöhung der Akzeptanz von Umweltschutzmaßnahmen 87

2. Erhöhung des Umweltbewußtseins $\quad 88$

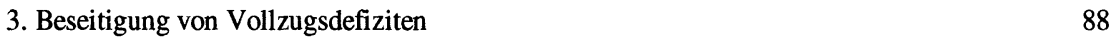


$\begin{array}{ll}\text { II. Modernisierung des Verwaltungsrechts } & 91\end{array}$

1. Beeinflussung anstelle Anordnung $\quad 91$

2. Entsubjektivierung des Verwaltungsrechts $\quad 93$

III. Wirtschaftsbezüge des Verwaltungsrechts $\quad 94$

3. Kapitel Der Inhalt des Anspruchs auf Umweltinformationen 96

I. Informationen als Gegenstand des Anspruchs $\quad 96$

1. Information als offener Begriff 96

2. Bezug der Informationen $\quad 97$

a) Daten über den Zustand der Umweltbereiche $\quad 98$

b) Daten über Umweltbeeinträchtigungen $\quad 99$

c) Daten über Umweltschutzmaßnahmen $\quad 99$

$\begin{array}{lr}\text { II. Personenbeziehungen } & 101\end{array}$

$\begin{array}{ll}\text { 1. Beteiligte } & 101\end{array}$

a) Der Informationssuchende $\quad 101$

b) Die Behörde $\quad 105$

c) Der Betroffene $\quad 110$

2. Personenbeziehungen $\quad 110$

a) Verwaltung und Informationssuchender $\quad 110$

b) Verwaltung und Betroffener 113

c) Informationssuchender und Betroffener $\quad 114$

III. Probleme des Anspruchsinhalts nach dem UGB-KomE 115

4. Kapitel Verfahren des Umweltinformations- gesetzes 117

I. Der Antrag nach § 5 UIG $\quad 117$

II. Ausgabeform der Informationen $\quad 118$

$\begin{array}{ll}\text { III. Kosten } & 121\end{array}$

IV. Probleme des Verfahrens nach dem UGB-KomE 123

5. Kapitel Grenzen des Anspruchs 125

I. Der Schutz der öffentlichen Belange $\quad 125$

1. Schutz allgemein höherrangiger öffentlicher Interessen 127

2. Schutz des staatlichen Innenbereiches 132

3. Schutz umweltschützend wirkender Vertrauenstatbestände $\quad 134$

4. Probleme des Schutzes der öffentlichen Belange nach dem UGB-KomE 134

II. Der Schutz der allgemeinen privaten Belange $\quad 135$

1. Schutz personenbezogener Daten 136

2. Schutz des geistigen Eigentums, insbesondere der Urheberrechte 140

a) Geistiges Eigentum $\quad 140$

b) Urheberrechte 142

(1) Urheberpersönlichkeitsrecht 142

(2) Verwertungsrechte 143 
(3) Grenzen des Urheberschutzes $\quad 145$

3. Probleme des Schutzes der allgemeinen privaten Belange nach dem UGB-KomE $\quad 146$

III. Der Schutz des Betriebs- und Geschäftsgeheimnisses 147

1. Inhaltliche Bestimmung des Begriffes $\quad 148$

a) Im Zusammenhang mit einem Geschäftsbetrieb 151

b) Nur wenigen bekannt 152

c) Subjektiver Geheimhaltungswille $\quad 155$

d) Objektiv schutzwürdiges Geheimhaltungsinteresse 155

(1) Begriffsbestimmung $\quad 155$

(2) Bestehen einer Berechtigung 156

(3) Objektiver Schutz bei rechtswidrigen Geheimnissen $\quad 159$

2. Grundrechtliche Herleitung $\quad 160$

a) Eigentumsgrundrecht Art. 14 GG 161

b) Berufsfreiheit Art. 12 GG 162

3. Die Befugnis zur Offenbarung der Geheimnisse als Brennpunkt des Konfliktes 163

a) Zustimmung 164

b) Gesetzliche Vorgaben 165

c) Güterabwägung $\quad 165$

(1) Gründe für das Informationsinteresse 165

(2) Gründe für Geheimhaltungsinteresse 167

(3) Grundzüge der Abwägung 167

(4) Verwendung von Negativ- oder Positivlisten 169

4. Vermeidung einseitiger Entscheidungen durch alternative Konfliktlösungen 170

a) Auszugsweise Übermittlung 171

b) Zusammenfassungen $\quad 172$

c) Ungenaue Angaben $\quad 174$

d) Anwaltslösung $\quad 174$

IV. Probleme des Betriebs- und Geschäftsgeheimnisses nach dem UGB-KomE 175

$\begin{array}{ll}\text { Zusammenfassung } & 178\end{array}$ 


\section{Literaturverzeichnis}

Anschütz, Gustav/Thoma, Richard (Hrsg.), Handbuch des deutschen Staatsrecht, Bd. 1, Tübingen 1930

Arzt, Clemens, Entwurf eines Umweltinformationsgesetzes vorgelegt, ZRP 1993, $18 \mathrm{ff}$.

Askelöf, Thomas / Fernemann Heugren, Ragna, Akteneinsicht in Schweden, in: Winter, Gerd

(Hrsg.), Öffentlichkeit von Umweltinformationen, Baden-Baden 1990, S. 473 ff.

Auernhammer, Herbert, Bundesdatenschutzgesetz, 3. Auflage, Köln, München 1993

Bachmann, Ulrich, Zum Behördenbegriff des Umweltinformationsgesetzes, GewArch 1995, 241 ff.

Badura, Peter, Der Eigentumsschutz des eingerichteten und ausgeübten Gewerbebetriebs, AöR 98 (1973), $153 \mathrm{ff}$.

Bauer, Hartmut, Geschichtliche Grundlagen der Lehre vom subjektiv-öffentlichen Recht, Berlin 1986

-, $\quad$ Altes und Neues zur Schutznormtheorie, AöR 113 (1988), 582 ff.

Baumbach, Wolfgang / Hefermehl, Adolf, Wettbewerbsrecht, 19. Auflage, München 1996

Benkard, Georg, Patentgesetz, Gebrauchsmustergesetz, 9. Auflage, München 1993

Berg, Wilfried, Der Schutz von Betriebs- und Geschäftsgeheimnissen im öffentlichen Recht unter besonderer Berücksichtigung des Umweltinformationsgesetzes, GewArch 1996, $177 \mathrm{ff}$.

Bieber, Roland, Informationsrechte Dritter im Verwaltungsverfahren, Ansätze zur legislativen und dogmatischen Neubewertung, DÖV 1991, 857 ff.

Bleckmann, Albert, Zu den Auslegungsmethoden des Europäischen Gerichtshofs, NJW 1982, $1177 \mathrm{ff}$.

Blumenberg, Hildegard, Die Umwelt-Informations-Richtlinie der EG und ihre Umsetzung in das deutsche Recht, NuR 1992, 8 ff.

Boc, Jan / Jendroska, Jerzy/Nowacki, Konrad, Akteneinsicht in Polen, in: Winter, Gerd (Hrsg.), Öffentlichkeit von Umweltinformationen, Baden-Baden 1990, S. 419 ff.

Böckenförde, Ernst-Wolfgang, Die verfassungstheoretische Unterscheidung von Staat und Gesellschaft als Bedingung der individuellen Freiheit, Opladen 1973

-, Der Verfassungstyp der deutschen konstitutionellen Monarchie im 19. Jahrhundert, in Bökkenförde (Hrsg.), Moderne deutsche Verfassungsgeschichte, 1815 - 1914, 2. Auflage, Königstein/Ts. 1981

-, Grundrechte als Grundsatznormen. Zur gegenwärtigen Lage der Grundrechtsdogmatik, Der Staat 29 (1990), $1 \mathrm{ff}$.

Borgsmidt, Kirsten, Ein Verfahrensgesetz und Neugestaltungen im dänischen Verwaltungsrecht, DÖV 1988, 70 ff.

Breuer, Rüdiger, Schutz von Betriebs- und Geschäftsgeheimnissen im Umweltrecht, NVwZ 1986, $171 \mathrm{ff}$. 
Breuer, Rüdiger, Umweltschutzrecht, in: Schmidt-Aßmann, Eberhard (Hrsg.), Besonderes Verwaltungsrecht, 10. Auflage, Berlin 1995

Bühler, Ottmar, Die subjektiven öffentlichen Rechte und ihr Schutz in der deutschen Verwaltungsrechtsprechung, Berlin, Stuttgart, Leipzig 1914

Bull, Hans-Peter, Datenschutz als Informationsrecht und Gefahrenabwehr, NJW 1979, $1178 \mathrm{ff}$.

Büllesbach, Alfred, Das neue Bundesdatenschutzgesetz, NJW 1991, 2593 ff.

Burkert, Herbert, Akteneinsicht und Datenschutz in Kanada, in: Winter, Gerd (Hrsg.), Öffentlichkeit von Umweltinformationen, Baden-Baden 1990, S. $283 \mathrm{ff}$.

-, Informationsrechte in Europa, in: Heymann, Thomas (Hrsg.), Informationsmarkt und Informationsschutz in Europa, Köln 1995, S. 86 ff.

Burmeister, Joachim H., Akteneinsicht in Großbritannien, in: Winter, Gerd (Hrsg.), Öffentlichkeit von Umweltinformationen, Baden-Baden 1990, S. $211 \mathrm{ff}$.

Conradi, Joachim, Das Öffentlichkeitsprinzip in der schwedischen Verwaltung, Berlin 1968

Cosack,Tilman/Tomerius, Stephan, Betrieblicher Geheimnisschutz und Interessen des Bürgers an Umweltinformationen bei der Aktenvorlage im Verwaltungsprozeß, NVwZ 1993, $841 \mathrm{ff}$.

Di Fabio, Udo, Informationen als hoheitliches Gestaltungsmittel, JuS 1997, 1 ff.

Dreier, Horst (Hrsg.), Grundgesetz-Kommentar, Bd. 1, Tübingen 1996

Drews, Bill/Wacke, Gerhard / Vogel, Klaus / Martens, Wolfgang, Gefahrenabwehr, 9. Auflage, Köln, München 1986

Druey, Jean Nicolas, Informationen als Gegenstand des Rechts, Zürich 1995

Dubach, Alexander, Das Recht auf Akteneinsicht, Zürich 1990

Düwel, Peter, Das Amtsgeheimnis, Berlin 1965

Eifert, Martin, Umweltinformation als Regelungsinstrument, DÖV 1994, 544 ff.

Eilers, Stephan / Schröer, Thomas, Der Schutz der betrieblichen Informationssphäre im Umweltinformationsgesetz, BB 1993, $1025 \mathrm{ff}$.

Ellwein, Thomas / Hesse, Joachim Jens (Hrsg.), Regierungssystem der Bundesrepublik Deutschland, 6. Auflage, Opladen 1983

Engel, Albert, Die rechtliche Aufarbeitung der Stasi-Unterlagen auf der Grundlage des StUG, Berlin 1995

Engel, Andreas, (Hrsg.), Umweltinformationssysteme in der öffentlichen Verwaltung, Heidelberg 1994

Engel, Rüdiger, Akteneinsicht und Recht auf Informationen über umweltbezogene Daten, Pfaffenweiler 1993

Erbguth, Wilfried/Stollmann, Frank, Zum Entwurf eines Umweltinformationsgesetzes, UPR 1994, $81 \mathrm{ff}$.

Erichsen, Hans-Uwe, Das Recht auf freien Zugang, NVwZ 1992, $409 \mathrm{ff}$. 
Erichsen, Hans-Uwe, Zur Umsetzung der Richtlinie des Rates über den freien Zugang an Informationen über die Umwelt: UBA-Bericht 1/92, Forschungsbericht 10106059, Berlin 1992

-, $\quad$ (Hrsg.), Allgemeines Verwaltungsrecht, 10. Auflage, Berlin, New York 1995

Everling, Ulrich, Durchführung und Umsetzung des Europäischen Gemeinschaftsrechts im Bereich des Umweltschutzes unter Berücksichtigung der Rechtsprechung des EuGH, NVwZ 1993, $209 \mathrm{ff}$.

Eyermann, Erich, Verwaltungsgerichtsordnung, 10. Auflage, München 1998

Faber, Angela, Die Bedeutung des Umweltinformationgesetzes in der Kommunalverwaltung, DVBl. 1995, $722 \mathrm{ff}$.

Feldhaus, Gerhard, Bundesimmissionsschutzrecht, Kommentar, 7 Bände, Lsbl., Heidelberg

Fluck, Jürgen, Der Schutz von Unternehmensdaten im Umweltinformationsgesetz, NVwZ 1994, $1048 \mathrm{ff}$.

- / Theuer, Andreas, Umweltinformationsrecht UIG, Lsbl., Heidelberg

- / Lemp, Ralph / Meyer-Rutz, Eckart (Hrsg.), Freier Zugang zu Umweltinformationen, Heidelberg 1992

Forsthoff, Ernst, Der Staat der Industriegesellschaft, 2. Auflage, München 1971

Fromm, Friedrich Karl / Nordemann, Wilhelm, Urheberrecht, 7. Auflage, Stuttgart, Berlin, Köln 1988

Gallwas, Hans-Ulrich, Der allgemeine Konflikt zwischen dem Recht auf informationelle Selbstbestimmung und der Informationsfreiheit, NJW 1992, $2785 \mathrm{ff}$.

Gamm, Otto-Friedrich Freiherr von, Urheberrecht, München 1968

Gaul, Dieter, Der erfolgreiche Schutz von Betriebs- und Geschäftsgeheimnissen, Neuwied 1994

Geiger, Rudolf, EG-Vertrag, Kommentar zu dem Vertrag zur Gründung der Europäischen Gemeinschaft, München 1993

Gornig, Gilbert-Hanno, Äußerungsfreiheit und Informationsfreiheit der Menschenrechte, Berlin 1988

Görres Gesellschaft zur Pflege der Wissenschaft im Katholischen Deutschland (Hrsg.), Staatslexikon, 7 Bände, Freiburg, Basel, Wien 1993

Graffe, Udo, Die Beteiligung des Bürgers an umweltschutzrechtlich relevanten Verfahren unter besonderer Berücksichtigung des VwVfG, Diss., Mainz 1980

Groeben, Hans von der/Thiesing, Jochen / Ehlermann, Claus-Dieter, Kommentar zum EU-/EGVertrag, 5. Auflage, Baden-Baden 1997

Grümmig, Klaus, Auskunftsansprüche des Bürgers - Auskunftspflichten in der freien Wirtschaft und der öffentlichen Verwaltung, VR 1991, 8 ff.

Gurlit, Elke Verwaltungsöffentlichkeit im Umweltrecht, Düsseldorf 1989

-, $\quad$ Akteneinsicht in den Vereinigten Staaten, in: Winter, Gerd (Hrsg.), Öffentlichkeit von Umweltinformationen, Baden-Baden 1990, S. 511 ff. 
Häberle, Peter, Grundrechte im Leistungsstaat, VVDStRL 30 (1972), S. 43 ff.

-, Verfassungsprinzipien ,im“ Verwaltungsverfahrensgesetz, in: Schmitt Glaeser, Walter (Hrsg.), Verwaltungsverfahren, Festschrift für Boorberg Verlag (1977), Stuttgart 1977, S. $71 \mathrm{ff}$.

Hahn, Werner, Offenbarungspflichten im Umweltrecht, Köln, München 1984

Hammerl, Christian, Der gläserne Betrieb, ZIP 1994, 1230 ff.

Hauck, Ernst, Wirtschaftsgeheimnisse - Informationseigentum kraft richterlicher Rechtsfortbildung?, Berlin 1987

Haueisen, Fritz, Akteneinsicht im Verwaltungsverfahren, NJW 1967, $2291 \mathrm{ff}$.

Hegele, Dorothea / Röger, Ralf, Umweltschutz durch Umweltinformationen - Chancen und Grenzen des neuen Informationsanspruchs, Berlin 1993

Hendler, Reinhard, Anmerkungen (zum Urteil des BVerwG vom 6.12.1996), JZ 1998, 245 f.

Henle, Victor, Die Masse im Massenverfahren, BayVBl. 1981, 1 ff.

Hesse, Konrad, Bemerkungen zur heutigen Problematik und Tragweite der Unterscheidung von Staat und Gesellschaft, DÖV 1975, 437 ff.

-, $\quad$ Grundzüge des Verfassungsrechts der Bundesrepublik Deutschland, 18. Auflage, Heidelberg 1991

Hirschberger, Winfried, Zugang des Bürgers zu staatlichen Informationen, Diss., Speyer 1983

Hoffmann-Riem, Wolfgang / Schmidt-Aßmann, Eberhard (Hrsg.), Reform des Allgemeinen Verwaltungrechts, Baden-Baden 1993

Hoppe, Werner/Beckmann, Martin, Umweltrecht, München 1989

- / Appold, Wolfgang, Gesetz über die Umweltverträglichkeitsprüfung, Köln, München 1995

Isensee, Josef / Kirchhof, Paul (Hrsg.), Handbuch des Staatsrechts der Bundesrepublik Deutschland, 9 Bände, Heidelberg 1997

Jaeger, Melwig F., Trade Secrets Law Handbook, NewYork 1984

Jarass, Hans, BImSchG, 2. Auflage, München 1993

- / Kloepfer, Michael / Kunig, Philip / Papier, Hans-Jürgen / Peine, Franz-Joseph / Rehbinder, Eckard/Salzwedel, Jürgen/Schmidt-Aßmann, Eberhard, Umweltgesetzbuch - Besonderer Teil (UGB-BT), Berichte des Bundesumweltamtes 4/94, Berlin 1994

- / Pieroth, Bodo, Grundgesetz für die Bundesrepublik Deutschland, 4. Auflage, München 1997

Jestaedt, Matthias, Der ,richtige“ Beklagte?, NWVBI 1989, 45 ff.

Kahl, Wolfgang, Der europarechtlich determinierte Verfassungswandel im Kommunikations- und Informationsstaat Bundesrepublik Deutschland, in: Haratsch, Andreas / Kugelmann, Dieter / Repkewitz, Ulrich (Hrsg.), Herausforderungen an das Recht der Informationsgesellschaft, Stuttgart, München, Hannover 1996, S. 8 ff.

Kavekordes, Johannes, Zur Rechtsstellung des Beigeladenen im Kartellrecht, WuW 1987, 365 ff. 
Kiminich, Otto / Lersner, Heinrich Frhr. v. / Storm, Peter-Christoph (Hrsg.), Handwörterbuch des Umweltrechts, 2. Auflage, Berlin 1994

Klein, Hans, Die Grundrechte im demokratischen Staat, Stuttgart 1974

Kloepfer, Michael, Umweltrecht, München 1989

-, $\quad$ Umweltinformationen durch Unternehmen, NuR 1993, $353 \mathrm{ff}$.

- /Rehbinder, Eckhard/ Schmidt-Aßmann, Eberhard / Kunig, Philip, Umweltgesetzbuch - Allgemeiner Teil (UGB-AT), Berichte des Bundesumweltamtes 7/90, Berlin 1990

- / Durner, Wolfgang, Der Umweltgesetzbuch-Entwurf der Sachverständigenkommission (UGBKomE), DVBl. 1997, 1081 ff.

Knack, Hans Joachim, Verwaltungsverfahrensgesetz, 5. Auflage, Köln, Berlin, Bonn 1996

Kneifel, Rainer, Freedom of information in den USA, CR 1990, $134 \mathrm{ff}$.

Knemeyer, Franz-Ludwig, Geheimhaltungsanspruch und Offenbarungsbefugnis in Verwaltungsverfahren, NJW 1984, 2241 ff.

-, $\quad$ Auskunftsanspruch und behördliche Auskunftsverweigerung, JZ 1992, 348 ff.

-, Die Wahrung von Betriebs- und Geschäftsgeheimnissen bei behördlichen Umweltinformationen. Zum Zielkonflikt von Geheimhaltungs- und Informationsinteresse im Umweltschutz, DB 1993, $721 \mathrm{ff}$.

-, $\quad$ Polizei- und Ordnungsrecht, 6. Auflage, München 1995

Knill, Christoph / Héritier, Adrienne, Neue Instrumente in der europäischen Umweltpolitik: Strategien für eine effektivere Implementation, in: Lübbe-Wolff, Gertrude (Hrsg.), Der Vollzug des europäischen Umweltrechts, Berlin 1996, S. 209 ff.

Knorr, Gerhard, Geheimhaltung und Gewerbeaufsicht, Berlin 1982

Koch, Hans-Joachim / Scheuing, Dieter (Hrsg.), Gemeinschaftskommentar zum Bundesimmissionsschutzgesetz (GK-BImSchG), Lsbl., Düsseldorf

Koenig, Christian, Möglichkeiten und Grenzen von Zertifikationsmärkten als Steuerungsmedien im Umweltrecht, DÖV 1996, 943 ff.

Kopp, Ferdinand, Der Beteiligtenbegriff des Verwaltungsverfahrensrechts in: Schmitt Glaeser, Walter (Hrsg.), Verwaltungsverfahren, Festschrift für Boorberg Verlag (1977), Stuttgart 1977, S. $180 \mathrm{ff}$.

-, Verwaltungsverfahrensgesetz, 6. Auflage, München 1996

- / Schenke, Wolf-Rüdiger, Verwaltungsgerichtsordnung, 11. Auflage, München 1998

Kragler, Peter, Schutz des geheimen Know-how, Landsberg/Lech 1987

Krämer, Ludwig, Defizite im Vollzug des EG-Umweltrechts und ihre Ursachen, in: Lübbe-Wolff, Gertrude (Hrsg.), Der Vollzug des europäischen Umweltrechts, Berlin 1996, S. 14 ff.

Kramer, Rainer, Umweltinformationsgesetz, Öko-Audit-Verordnung, Umweltzeichenverordnung, Stuttgart, Berlin, Köln 1994 
Kunig, Philip, Das Recht des öffentlichen Dienstes, in: Schmidt-Aßmann, Eberhard (Hrsg.), Besonderes Verwaltungsrecht, 10. Auflage, Berlin, New York 1995

Ladeur, Karl-Heinz, Akteneinsicht in Italien, in: Winter,Gerd (Hrsg.), Öffentlichkeit von Umweltinformationen, Baden-Baden 1990, S. $249 \mathrm{ff}$.

LAI, Beschluß des Länderausschusses für Immissionsschutz über Auskunftsrechte und Auskuftspflichten gegenüber Nachbarn bei Emissionen von Industrieanlagen, NVwZ 1986, 283 ff.

Landmann, Robert von /Rohmer, Gustav, Umweltrecht, 3 Bände, Lsbl., München

Larenz, Karl, Allgemeiner Teil des Deutschen Bürgerlichen Rechts, 7. Auflage, München 1989

-, $\quad$ Methodenlehre der Rechtswissenschaft, 6. Auflage, Berlin 1991

Laubinger, Hans-Werner, Grundrechtsschutz durch Gestaltung des Verwaltungsverfahrens, VerwArch 73 (1982), $60 \mathrm{ff}$.

Lodde, Stephan, Informationsrechte des Bürgers gegen den Staat, Köln, Berlin, Bonn 1996

Lübbe-Wolff, Gertrude, Das niederländische Gesetz über die Verwaltungsöffentlichkeit, Die Verwaltung 13 (1980), $339 \mathrm{ff}$.

-, Das Kooperationsprinzip im Umweltrecht - Rechtsgrundsatz oder Deckmantel des Vollzugsdefizits, NuR 1989, 295 ff.

-, $\quad$ Öko-Audit und Deregulierung, ZUR 1996, 173 ff.

-, (Hrsg.), Der Vollzug des europäischen Umweltrechts, Berlin 1996

Lutter, Marcus, Die Auslegung angeglichenen Rechts, JZ 1992, 593 ff.

Mahrer, Isabell, Die Öffentlichkeit amtlicher Stellen in Schweden, ZSR NF 88 (1969), I, 317 ff.

Martens, Wolfgang, Öffentlichkeit als Rechtsbegriff, Bad Homburg, Berlin, Zürich 1969

Maunz, Theodor / Dürig, Günter, Grundgesetz, 4 Bände, Lsbl., München

Maurer, Hartmut, Allgemeines Verwaltungsrecht, 10. Auflage, München 1995

Meininger, Frank, Die EG-Umweltinformationsrichtlinie in der Verwaltungspraxis, NVwZ 1994, $150 \mathrm{ff}$.

Mengel, Hans Joachim, Akteneinsicht in Verwaltungsverfahren, Die Verwaltung 23 (1990), $377 \mathrm{ff}$.

Meyer, Hans / Borgs-Maciejewski, Hermann, Verwaltungsverfahrensgesetz, 2. Auflage, Frankfurt/Main 1982

Münch, Ingo von / Kunig, Philip, Grundgesetz-Kommentar, 4. Auflage, München 1992

Noll, Peter, Gesetzgebungslehre, Reinbeck bei Hamburg 1973

Obermayer, Klaus, Kommentar zum Verwaltungsverfahrensgesetz, 2. Auflage, Neuwied 1990

Odersky, Walter, Harmonisierende Auslegung und europäische Rechtskultur, ZEuP 1994, 1 ff.

Oppermann, Thomas, Europarecht, München 1991

Ossenbühl, Fritz, Umweltschutz und Gemeinwohl in der Rechtsordnung, VR 1983, 300 ff. = Bitburger Gespräche Jb. 1983, S. 5 ff. 
Ossenbühl, Fritz, Informelles Hoheitshandeln im Gesundheits- und Umweltschutz, in: Jahrbuch des Umwelt- und Technikrechts Bd. 3, Düsseldorf 1987, S. 27 ff.

Palandt, Otto, BGB, 57. Auflage, München 1998

Peters, Hans-Joachim, Das Recht der Umweltverträglichkeitsprüfung, UVPG, Bd. 2, Baden-Baden 1996

Pétren, Gustav, Die Aktenöffentlichkeit in Schweden, VerwArch 1958, 323 ff.

Pieroth, Bodo, Die planende Verwaltung zwischen Geheimhaltung und Öffentlichkeit, JuS 1981, $625 \mathrm{ff}$.

Pipkorn, Jörn, Auskunftsansprüche gegenüber Verwaltungsbehörden, DÖV 1970, $171 \mathrm{ff}$.

Pitschas, Rainer, Allgemeines Verwaltungsrecht als Teil der öffentlichen Informationsordnung, in: Hoffmann-Riem, Wolfgang / Schmidt-Aßmann, Eberhard (Hrsg.), Reform des Allgemeinen Verwaltungsrechts, Baden-Baden 1993, S. 219 ff.

Rebentisch, Manfred, Probleme des Geheimnisschutzes im Rahmen der Emissionserklärung, NJW 1980, 99 ff.

Rehbinder, Eckard / Kayser, Deltev / Klein, Helmuth, Chemikaliengesetz, Heidelberg 1985

Reinhardt, Michael, Umweltinformationen als subjektives Recht, Die Verwaltung 30 (1997), $161 \mathrm{ff}$.

Rhein, Cornelius, Das Gemeinschaftssystem für das Umweltmanagement und die Umweltbetriebsprüfung: Ein neues Instrument des Umweltschutzes im Gemeinschaftsrecht und deutschen Recht, Nomos, Baden-Baden 1996

Richler, Werner, Schutz von Betriebs- und Geschäftsgeheimnissen im Umweltrecht, Diss., Regensburg 1989

Röger, Ralf, Ein neuer Informationsanspruch auf europäischer Ebene: Der Verhaltenskodex vom 6.12.1993 für den Zugang der Öffentlichkeit zu Kommissions- und Ratsdokumenten, DVBl. 1994, 1182 ff.

-, $\quad$ Umweltinformationsgesetz, Köln, Berlin, Bonn 1995

-, Das Recht des Antragstellers auf Wahl des Informationszugangs im Rahmen der Ermessensentscheidung nach § 4 I S. 2 UIG, DVBl. 1997, 885 ff.

Rosenstiel, Anja v., Ökologie - ein attraktives Geschäftsfeld für Juristen, NJW-CoR 1997, $210 \mathrm{ff}$.

Rosler, Gerhard, Informationsfreiheit in den Vereinigten Staaten von Amerika, Heidelberg 1984

Salje, Peter, Umwelthaftungsgesetz, München 1993

Scherer, Joachim, Verwaltung und Öffentlichkeit, Baden-Baden 1978

-, $\quad$ Datenschutz und Datenzugang, JZ 1979, 389 ff.

Scherzberg, Arno, Der freie Zugang zu Informationen über die Umwelt - Rechtsfragen zur Richtlinie 90/313/EWG, UPR 1992, 48 ff.

-, Freedom of information - deutsch gewendet: Das neue Umweltinformationsgesetz, DVB1. 1994, $733 \mathrm{ff}$. 
Scheuner, Ulrich, Das repräsentative Prinzip in der modernen Demokratie, in: Staatstheorie und Staatsrecht, Berlin 1978, S. 245 ff.

Schimanski, Werner, Aktenvorlage, Akteneinsicht und Datenschutz, Soziale Sicherheit 1987, $207 \mathrm{ff}$.

Schlachter, Jörg, Mehr Öffentlichkeit wagen, Heidelberg 1993

Schmid, Paul, Der gesetzliche Schutz der Fabrik- und Geschäftsgeheimnisse, Tübingen 1907

Schmidt, Dietmar / Dörr, Erwin, Stasi-Unterlagen-Gesetz, Köln 1993

Schmidt, Karsten, Drittschutz, Akteneinsicht und Geheimnisschutz im Kartellverfahren, Köln, München 1992

Schmidt-Aßmann, Eberhard, Verwaltungsverantwortung und Verwaltungsgerichtsbarkeit, VVDStRL 34 (1976), S. 221 ff.

-, (Hrsg.), Besonderes Verwaltungsrecht, 10. Auflage, Berlin, New York 1995

Schnur, Roman, Pressefreiheit, VVDStRL 22 (1965), S. $101 \mathrm{ff}$.

Schoch, Friedrich, Staatliche Informationspolitik und Berufsfreiheit, DVB1. 1991, $667 \mathrm{ff}$.

-, Öffentlich-rechtliche Rahmenbedingungen einer Informationsordnung, VVDStRL 57 (1998), S. $158 \mathrm{ff}$.

- /Schmidt-Aßmann, Eberhard/Pietzner, Rainer, Verwaltungsgerichtsordnung, Lsbl., München

Scholz, Rupert / Pitschas, Rainer, Informationelle Selbstbestimmung und staatliche Informationsordnung, Berlin 1984

Schomerus, Thomas, Anspruchsvoraussetzungen und Verwaltungsverfahren nach dem Umweltinformationsgesetz. Zum Umgang mit betriebsbezogenen Umweltdaten, ZUR 1994, $226 \mathrm{ff}$.

- / Schrader, Christian / Wegener, Bernhard, Umweltinformationsgesetz, Baden/Baden 1995

Schrader, Christian, Kostenerhebungen für den Zugang zu Umweltinformationen - Nationales Recht im Konflikt mit europarechtlichen Vorgaben, ZUR 1994, 221 ff.

-, Anmerkungen (zu dem Beschluß des OVG Schleswig-Holstein vom 10.7.1996), ZUR 1996, $98 \mathrm{ff}$.

Schröder, Meinhardt, Staatsrechtliche Aspekte einer Aktenöffentlichkeit im Verwaltungsverfahren, Die Verwaltung 4 (1971), $301 \mathrm{ff}$.

-, Geheimhaltungsschutz im Recht der Umweltchemikalien, Bericht des Umweltbundesamtes, 10/80, Berlin 1980

-, $\quad$ Der Schutz von Betriebs- und Geschäftsgeheimnissen, UPR 1985, 394 ff.

-, Auskunft und Zugang in bezug auf Umweltdaten als Rechtsproblem, NVwZ 1990, $905 \mathrm{ff}$.

Schuhmacher, Alex, Das Daten- und Informationssystem des MURL (DIM). Ein benutzerfreundlicher Zugang zu Umweltdaten, in: Engel, Andreas (Hrsg.), Umweltinformationssysteme in der öffentlichen Verwaltung, Heidelberg 1994, S. 49 ff.

Schulte, Martin, Schlichtes Verwaltungshandeln, Tübingen 1995 
Schulze-Fielitz, Helmuth, Kooperatives Recht im Spannungsfeld von Rechtsstaatsprinzip und Verfahrensökonomie, DVBl. 1994, 657 ff.

Schwab, Siegfried, Das Recht auf Akteneinsicht, DÖD 1997, 145 ff.

Schwanenflügel, Matthias von, Das Öffentlichkeitsprinzip des EG-Umweltrechts. Zur Bedeutung der Richtlinie über den freien Zugang zu Umweltinformationen, DVB1. 1991, $93 \mathrm{ff}$.

-, Die Richtlinie über den freien Zugang zu Umweltinformationen als Chance für den Umweltschutz, DÖV 1993, 95 ff.

Sening, Christoph, Abschied von der Schutznormtheorie im Naturschutzrecht, NuR 1980, $102 \mathrm{ff}$.

Siedler, Frank/Zeitler, Herbert, Bayerisches Straßen- und Wegegesetz, 2. Auflage, München 1972

Simitis, Spiros, Kommentar zum Bundesdatenschutzgesetz, 4. Auflage, Lsbl., Baden-Baden

- / Dammann, Ulrich / Mallmann, Otto / Reh, Hans-Joachim, Dokumentation zum Bundesdatenschutzgesetz, Lsbl., Baden-Baden

Spanner, Hans, Ein Entwurf eines Verwaltungsverfahrensgesetz, DVB1. 1964, $845 \mathrm{ff}$.

Starck, Christian, Rechtsvergleichung im öffentlichen Recht, JZ 1997, 1021 ff.

Stelkens, Paul / Bonk, Heinz/Sachs, Michael, Verwaltungsverfahrensgesetz, 3. Auflage, München 1990

Stettner, Rupert, Information als Verfassungsgut, in: Merten, Detlef / Schmidt, Reiner / Stettner, Rupert (Hrsg.), Der Verwaltungsstaat im Wandel, Festschrift für Franz Knöpfle zum 70. Geburtstag, München 1996, S. 351 ff.

Stich, Rudolph / Porger, Karl-Wilhelm, Bundesimmissionsschutzgesetz, Lsbl., Stuttgart

Stober, Rolf, Zur Bedeutung des Einwendungsschlusses im atom- und immissionsschutzrechtlichen Verfahren, AöR 106 (1981), 41 ff.

Stoltenberg, Klaus, Stasiunterlagengesetz, Baden-Baden 1992

-, Die historische Entscheidung für die Öffnung der Stasi-Akten - Anmerkungen zum StUG, DtZ 1992, 65 ff.

-, $\quad$ Zu den Änderungen des Stasi-Unterlagen-Gesetzes, DtZ 1994, 386 ff.

Stürner, Rolf, Die gewerbliche Geheimsphäre im Zivilprozeß, JZ 1985, 453 ff.

Taeger, Jürgen, Die Offenbarung von Betriebs- und Geschäftsgeheimnissen, Baden-Baden 1988

Theuer, Andreas, Der Zugang zu Umweltinformationen aufgrund des Umweltinformationsgesetzes (UIG), NVwZ 1996, 326 ff.

Trute, Hans-Heinrich, Die Regelung des Umgangs mit den Stasi-Unterlagen im Spannungsfeld von allgemeinem Persönlichkeitsrecht und legitimen Verwendungszwecken, JZ 1992, 1043 ff.

Turiaux, André, Das neue Umweltinformationsgesetz, NJW 1994, 2319 ff.

-, Umweltinformationsgesetz, München 1995

Ule, Carl, Verwaltungsprozeßrecht, 9. Auflage, München 1987 
Ule, Carl / Becker, Franz, Verwaltungsverfahren im Rechtsstaat, Köln, Berlin 1964

- /Laubinger, Hans-Werner, Verwaltungsverfahrensrecht, 3. Auflage, Köln, Berlin, Bonn 1986

Vahldiek, Carola, Neue Entwicklungen in der Rechtsprechung zum Umweltinformationsrecht, ZUR 1997, 144 ff.

-, $\quad$ Anmerkungen zur Entscheidung des EuGH vom 17.6.1998, ZUR 98, 200 ff.

Wadle, Elmar, Geistiges Eigentum - Bausteine zur Rechtsgeschichte, Weinheim, New York, Basel 1996

Wagner, Hellmut, Effizienz des Ordnungsrechts für den Umweltschutz?, NVwZ 1995, 1046 ff.

Wassermann, Rudolf (Hrsg.), Alternativkommentar zum Grundgesetz (AK-GG), 2. Auflage, Neuwied, Darmstadt 1989

Weber, Max, Wirtschaft und Gesellschaft, Studienausgabe, 5. Auflage, Tübingen 1980

Wegener, Bernhard, Umsetzung der EG Richtlinie über den freien Zugang zu Umweltinformationen - Zum Referentenentwurf des BMU, IUR 1992, $211 \mathrm{ff}$.

-, Die unmittelbare Geltung der EG-Richtlinie über den freien Zugang zu Umweltinformationen, ZUR 1993, 17 ff.

Wickrath, Susan, Bürgerbeteiligung im Recht der Raumordnung und Landesplanung, Münster 1992

Wieckmann, Hans-Joachim, Akteneinsicht und Wahrung von Geschäftsgeheimnissen im Kartellverwaltungsverfahren, WuW 1983, $13 \mathrm{ff}$.

Winter, Gerd, Akteneinsicht in Frankreich, UPR 1989, $85 \mathrm{ff}$.

-, Was sind Umweltinformationen, was Vorverfahren im Sinne der Umweltinformationsrichtlinie?, NuR 1997, $335 \mathrm{ff}$.

-, (Hrsg.), Öffentlichkeit von Umweltinformationen, Baden-Baden 1990

Zenkert, Georg, Die Macht der öffentlichen Meinung, Der Staat 31 (1992) S. 321 ff.

\section{Anmerkungen zu Quellen im Internet:}

In folgender Untersuchung werden Verweise auf virtuelle Seiten im Internet vorgenommen. Damit wird der Aktualität des Themenbereichs „Informationszugang“ Rechnung getragen. Der Verweis auf Internet-Adressen findet nur statt, wenn Tatsachen aufgrund ihrer Aktualität nicht auf herkömmlichen Weise zur Verfügung stehen.

Es werden nur Adressen von Servern verwendet, die von öffentlichen Stellen von Staaten oder von den Europäischen Institutionen betrieben und betreut werden.

Im Anschluß an die Adresse findet sich ein Verweis auf den letzten erfolgreichen Zugriff.

Die Adressen dieser Seiten, die sog. URLs, werden in die Adressleiste des Internetzugangsprogrammes, dem sog. Browser, eingegeben. 